\title{
Design of electromagnetic sensor arrays optimised for inversion of the magnetic polarisability tensor
}

DOI:

10.1109/SAS.2015.7133653

Link to publication record in Manchester Research Explorer

\section{Citation for published version (APA):}

Marsh, L., Rehim, O. A. A., Tan, Y., O'Toole, M., Armitage, D., \& Peyton, A. (2015). Design of electromagnetic sensor arrays optimised for inversion of the magnetic polarisability tensor. In IEEE Sensors Applications Symposium (SAS), 2015 (pp. 4pp.). IEEE. https://doi.org/10.1109/SAS.2015.7133653

\section{Published in:}

IEEE Sensors Applications Symposium (SAS), 2015

\section{Citing this paper}

Please note that where the full-text provided on Manchester Research Explorer is the Author Accepted Manuscript or Proof version this may differ from the final Published version. If citing, it is advised that you check and use the publisher's definitive version.

\section{General rights}

Copyright and moral rights for the publications made accessible in the Research Explorer are retained by the authors and/or other copyright owners and it is a condition of accessing publications that users recognise and abide by the legal requirements associated with these rights.

\section{Takedown policy}

If you believe that this document breaches copyright please refer to the University of Manchester's Takedown Procedures [http://man.ac.uk/04Y6Bo] or contact uml.scholarlycommunications@manchester.ac.uk providing relevant details, so we can investigate your claim.

\section{OPEN ACCESS}




\title{
Design of Electromagnetic Sensor Arrays Optimised for Inversion of the Magnetic Polarisability Tensor
}

\author{
Liam A Marsh*, Member, IEEE, Omar A Abdel Rehim, Yee M Tan, Michael D O'Toole, \\ David W Armitage and Anthony J Peyton, Fellow, IEEE \\ School of Electrical and Electronic Engineering \\ The University of Manchester \\ Manchester, M13 9PL, UK \\ *Email: liam.marsh@manchester.ac.uk
}

\begin{abstract}
This paper presents a method for the simulation of sensitivity maps from an array of coils. Some of the criteria necessary for designing a coil array capable of inversion of the magnetic polarisability are examined, and sensitivity maps are analysed with this in mind. The summarised sensitivity map for a single optimised array is presented, as well as the results of noise testing of an inversion algorithm using simulated measurements. Finally, a method for the construction and testing of such an array is presented.
\end{abstract}

Keywords-electromagnetic induction, ERW detection, magnetic polarisability tensor, inversion

\section{INTRODUCTION}

Inductive metal detection has been on the front line of landmine detection for several decades. During this time deminers have relied upon the response of the metallic components present in landmines to identify buried or visually obscured targets. Whilst there is a large number of landmines which contain a significant amount of metallic components, there is also a significant number of so called 'minimum metal' mines, which contain almost no metal components [1]. This makes their detection extremely difficult, and therefore increases the already substantial risk involved in the demining process.

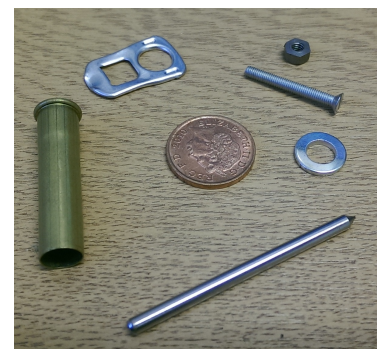

Fig. 1. Example items of metallic clutter

Landmines are often found in sites which are polluted with innocuous metallic items e.g. litter, shrapnel from previous conflicts or secondary mechanical threats such as caltrops [2], which mitigate the effectiveness of metal detector technologies. Due to the limitations of the current generation of inductive metal detectors, both landmines and innocuous metallic items exhibit the same response from the detector; this is typically an audible sound in a deminer's earpiece. Demining procedures dictate that careful excavation of the target area must take place after a positive identification with an inductive metal detector. Often the excavation reveals an object which poses no threat to the deminer; examples of such innocuous items are provided in figure 1. This frequent excavation of innocuous items significantly increases the time taken to clear a field of genuine threats and can lead to complacency by the deminer. As an example of this point, a 1994 report by the United Nations stated that in Cambodia there were 129 recorded innocuous metallic items excavated for every landmine [3], and in 2003 The Cambodia Daily reported that the situation had not significantly improved [4]. In the last few years there have been some recent advancements in detector technology through the combined use of inductive metal detection and ground penetrating radar (GPR) [5]. The use of GPR significantly aids deminers by adding a new method of target classification, however the technology has the potential to further reduce false positive mine detections through improved metal characterisation.

The UN estimates that it will take 1,100 years to clear the world of landmines using current technology, and assuming no further land mines are planted [6]; the estimated associated cost of this is \$33 billion (USD). The design of a system which is capable of identifying buried or visually obscured metallic items has the potential to significantly reduce the time required to clear a given area of threats. Our previous research has shown the possibility of inverting the magnetic polarisability tensor, $\overleftrightarrow{\mathbf{M}}$, and its use in classifying targets [7] [8].

This paper identifies the necessary criteria for coil arrays which allow for effective inversion of the magnetic polarisability tensor, and presents a method for evaluating the suitability of a given array for this task. A geometry is presented which is considered to be particularly suitable for this purpose, as are results for both forward and inverse modelling.

\section{BACKGROUND}

The magnetic polarisability tensor is a complex, symmetric, $3 \times 3$ matrix which varies with frequency. It is defined in the following way:

$$
\overleftrightarrow{\mathbf{M}}(f)=\left[\begin{array}{lll}
M_{11}^{\prime}+j M_{11}^{\prime \prime} & M_{12}^{\prime}+j M_{12}^{\prime \prime} & M_{13}^{\prime}+j M_{13}^{\prime \prime} \\
M_{12}^{\prime}+j M_{12}^{\prime \prime} & M_{22}^{\prime}+j M_{22}^{\prime \prime} & M_{23}^{\prime}+j M_{23}^{\prime \prime} \\
M_{13}^{\prime}+j M_{13}^{\prime \prime} & M_{23}^{\prime}+j M_{23}^{\prime \prime} & M_{33}^{\prime}+j M_{33}^{\prime \prime}
\end{array}\right]
$$

The above definition shows that at each frequency there are six unique complex terms in the tensor, defined as $M_{11}, M_{12}$, 
$M_{13}, M_{22}, M_{23}$ and $M_{33}$, each of which corresponds to the $\mathrm{xx}, \mathrm{xy}, \mathrm{xz}, \mathrm{yy}, \mathrm{yz}$ and $\mathrm{zz}$ sensitivity components respectively. In order for any inversion algorithm to be capable of determining the values of $\overleftrightarrow{\mathbf{M}}$ it is necessary to use a measurement system which is sensitive to each of these six components. Any system which lacks sensitivity in one, or more of these components will yield noisy or meaningless values in place of the relevant components. Consequently, it can be said the first requirement of any potential system designed to yield $\overleftrightarrow{\mathbf{M}}$ is that it must have good (and preferably even) sensitivity to all six dimensions of the corresponding values in the magnetic polarisability tensor. As a consequence of this point, it is necessary to define a method that allows for comparison of multi-dimensional sensitivity for each candidate coil array. This is discussed in more detail later in the paper.

In order to maximise receive channel gain and hence take full advantage of SNR it is also necessary for all receive coils to be nulled with respect to any transmit coils in the system. This is typically done by crossing over the turns on each receive coil at some defined mid-point so as to enclose zero net area in a symmetrical configuration with respect to the transmit coil(s). Such a configuration is referred to as a 'gradiometer' arrangement. This rejection of the primary (incident) field allows the dynamic range of any receive hardware to be fully-devoted to that of the imbalance caused as a result of the presence of a metallic object, rather than the transmitter coupling. Although it is theoretically possible to invert the magnetic polarisability tensor without rejecting the background signal, from the requirements of signal-to-noise ratio, and also due to practical limitations in dynamic range the only geometries which have been considered in this study are those which fulfil the zero net coupling criteria.

\section{COIL DESIGNS}

A large number of candidate coil arrays were simulated and compared in the pursuit of a coil array that could be considered to be optimised for the process of tensor inversion. Some examples of coil geometries which were investigated are shown in figure 2; as mentioned previously, all of these geometries have receive coils which are nulled with respect to any transmit coils. The most suitable coil array that was considered, and the array used for the subsequent analysis presented in this paper, is shown in figure 3. It consists of a single transmit coil of dimensions $0.3 \mathrm{~m}$ square, and four receive coils, each of which is half of the size of the transmit coil and is displaced and rotated $90^{\circ}$ about the z-axis.

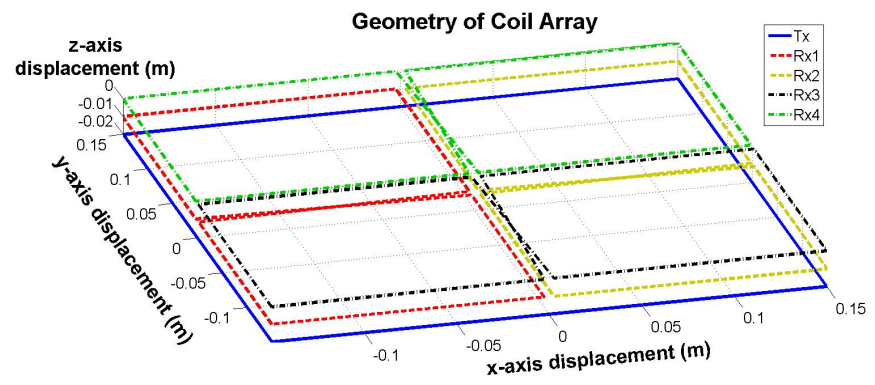

Fig. 3. Prototype coil array
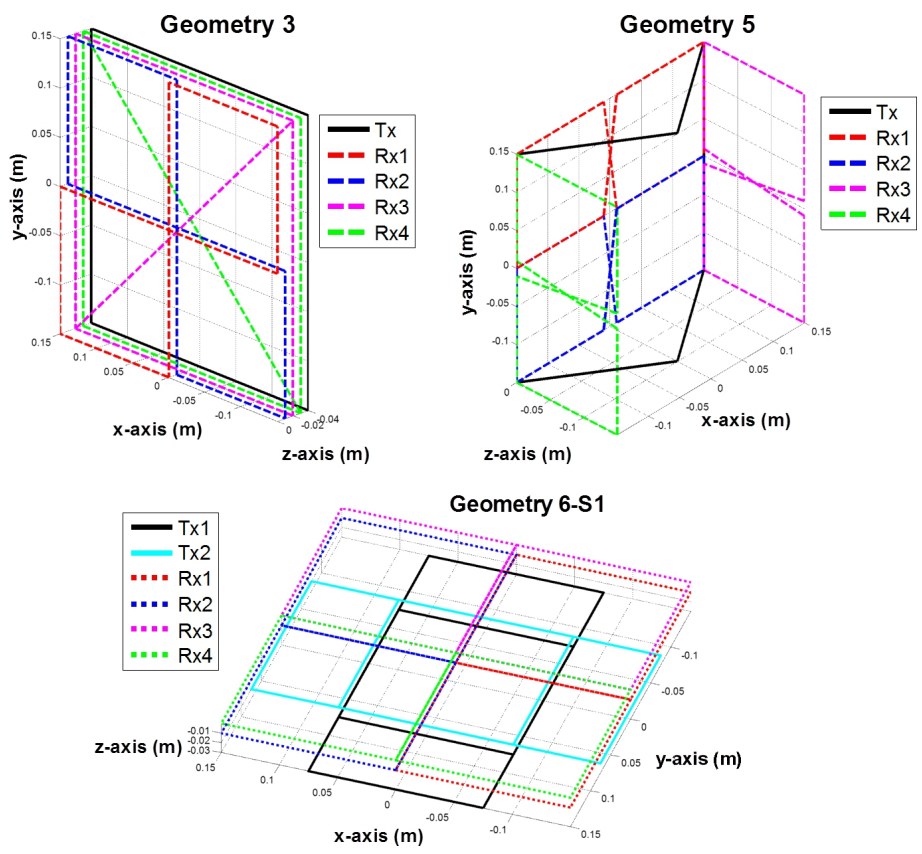

Fig. 2. Candidate coil arrays

\section{DESign Evaluation}

All candidate coil geometries were analysed by means of forward modelling. The sensitivity map for the forward model was produced according to equations 1 to 3 . This process is described further under 'Forward Modelling' below.

$\left[\begin{array}{lll}M_{11} & M_{12} & M_{13} \\ M_{12} & M_{22} & M_{23} \\ M_{13} & M_{23} & M_{33}\end{array}\right]\left[\begin{array}{l}H t x_{x} \\ H t x_{y} \\ H t x_{z}\end{array}\right]\left[\begin{array}{l}H r x_{x} \\ H r x_{y} \\ H r x_{z}\end{array}\right]=V$

The above can be rearranged in the following way:

$$
\begin{array}{r}
M_{11}\left(H t x_{x} H r x_{x}\right)+M_{12}\left(H t x_{x} H r x_{y}+H t x_{y} H r x_{x}\right)+ \\
M_{13}\left(H t x_{x} H r x_{z}+H t x_{z} H r x_{x}\right)+M_{22}\left(H t x_{y} H r x_{y}\right)+ \\
M_{23}\left(H t x_{y} H r x_{z}+H t x_{z} H r x_{y}\right)+M_{33}\left(H t x_{z} H r x_{z}\right)=V
\end{array}
$$

$$
\mathbf{A}\left[\begin{array}{llllll}
M_{11} & M_{12} & M_{13} & M_{22} & M_{23} & M_{33}
\end{array}\right]^{T}=V
$$

Only candidate coil arrays which met the necessary criteria for sensitivity in the forward modelling analysis were analysed by means of simulated inversion. In this paper, inverse modelling is only discussed for the candidate array show in figure 3. The algorithm used for this analysis is based upon inversion of equation 3 as shown in equation 4 . This was implemented in MATLAB using the minimisation function e04fy [9] in the NAG Toolbox [10].

$$
V \mathbf{A}^{-1}=\left[\begin{array}{llllll}
M_{11} & M_{12} & M_{13} & M_{22} & M_{23} & M_{33}
\end{array}\right]^{T}
$$




\section{A. Forward Modelling}

A method was devised to allow for straightforward comparison of different coil geometries. This method was based upon the comparison of the magnitude of the six sensitivity components in fixed $x-y$ planes at distances of $z=0.05$ $\mathrm{m}, 0.1 \mathrm{~m}$ and $0.2 \mathrm{~m}$. The desired result was to identify a coil array which possessed regions of high sensitivity for all six components, so that successful tensor inversion could be achieved. Due to the planar nature of the coil array it was expected that the components orthogonal to the plane should dominate all others; also due the the nature of magnetic fields it was not expected that any practically suitable design should exist which has a region of high sensitivity for all components in a single location. Demining practices allow for a scan across the entirety of the detector's sensitivity space which consists of many locations, which together represents all locations within the given plane of the sensitivity matrix. Consequently, regions of heightened sensitivity to each of the six sensitivity dimensions remain valid regardless of their location within the plane.

Figure 4 displays the summarised sensitivity profile of the geometry in figure 3 in the $x-y$ plane defined by $z=0.1 \mathrm{~m}$. The plot shows the summation of the magnitude of the sensitivitiy map for each coil pair, so that it is possible to visualise the sensitivity of the array in a single figure. The colour map is normalised as a percentage such that the value of $100 \%$ correcponds to the peak magnitude across all six plots. The normalisation factor is shown at the bottom of the figure.

In figure 4 it is possible to see that the sensitivity components which contain a $\mathrm{z}$ term are dominant; this is expected for a planar array of this type. The fact that the remaining terms show regions of non-zero sensitivity on the colour map when on the same scale as the $\mathrm{z}$ terms is what makes this coil array promising in terms of its suitability for inversion purposes. By comparing the relative peak magnitudes for each dimension of the sensitivity matrix, it is possible to identify that the worst case scenario is for the $\mathrm{xx}$, and yy components, each of which has a peak magnitude of approximately $27 \%$ of the global peak magnitude. Many of the other planar coil arrays that were considered were an order of magnitude lower than this. This was due to a combination of the strong dominance of the $\mathrm{z}$ terms, and an inherent insensitivity to the other $\mathrm{x}$ and $\mathrm{y}$ terms. Some testing was carried out with a modified version of the geometry shown in figure 3, which suppressed the z-dominance by means of the creation of a 'magnetic cavity'. To achieve this a second transmit coil, wound in opposition ot the first, was simulated in the centre of the original coil. Although the approach was successful in its aim of suppressing z-sensitivity dominance, the simulations showed that the results of the inversion were not significantly improved, and such an added increase in system complexity was unjustifiable.

\section{B. Inverse Modelling}

The inversion algorithm described previously was applied to a set of 100 simulated measurement sets, each of which used random tensor values constrained to the range of $0 \leq \bar{M}_{i j} \leq 2 \mathrm{~m}^{3}$. These sets consisted of measurements at 625 locations following a raster trajectory, as shown in figure 5. In the simulation the coils remained in a static location and

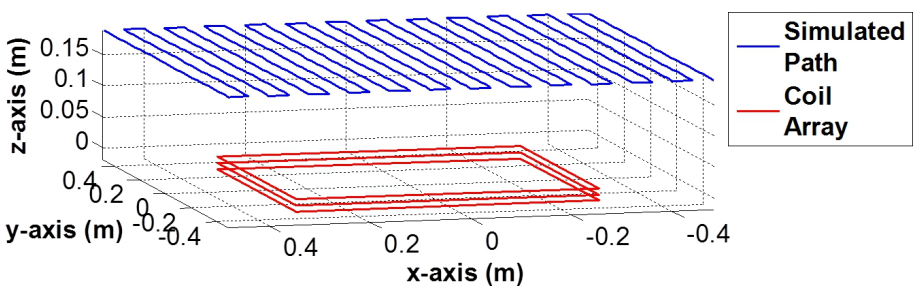

Fig. 5. Simulated path used for inversion testing

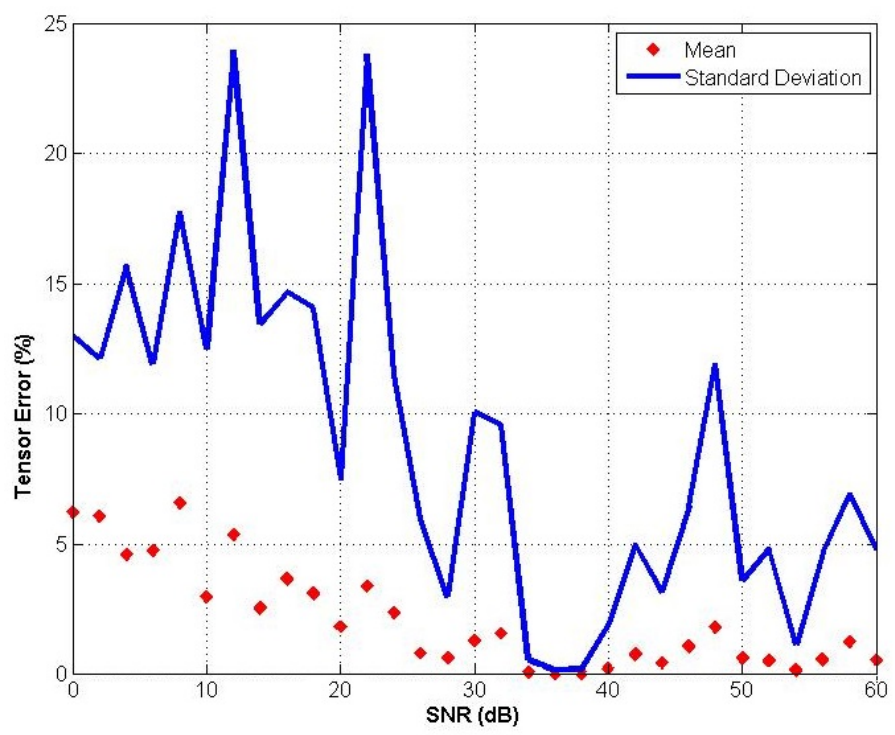

Fig. 6. Simulated inversion results for $\mathrm{SNR}=60 \mathrm{~dB}$ to $0 \mathrm{~dB}$, known trajectory with unknown offset

the object was swept; this process is analogous to sweeping the coils over the object. The entire measurement grid was offset to apply an error of $0 \leq \overrightarrow{\mathbf{L}} \leq 10 \mathrm{~cm}$. An initial guess for each measurement was calculated by adding a randomised displacement in the range $\pm 3 \mathrm{~cm}$ was then added to the original $\mathrm{x}, \mathrm{y}$ and $\mathrm{z}$ components of each point. The algorithm attempted to resolve the grid offset and invert the simulated tensor. The randomised tensors, location offsets and erroneous initial positional estimates for each of the 100 measurement sets were stored to allow for fair testing of the performance of the algorithm in the presence of noise.

Noise was added to the signal by means of synthesising in-band sinusoids with randomised phase offsets; out of band noise was not considered. The magnitude of the noise signals was varied to simulate SNR values from $60 \mathrm{~dB}$ to $0 \mathrm{~dB}$, at intervals of every $2 \mathrm{~dB}$. The average tensor error, as defined previously, is plotted on figure 6 for each SNR value.

\section{Construction}

The prototype coil former was designed using CAD and fabricated by means of 3D printing using polyamide 2200 [11]. Separate formers were produced for the transmit coil, and each half of the receive coils. These were then wound using enamel wire, and were fitted together using interlocking pegs which had been added to the 3D model. For increased durability the entire array was then encapsulated in resin. A photograph of the prototype array is shown in figure 7 . The inset figure shows 

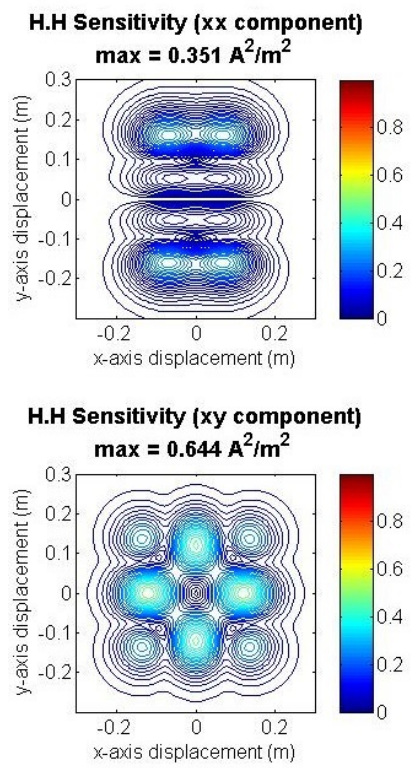

\section{$z=0.1 \mathrm{~m}, 21$ contour levels}
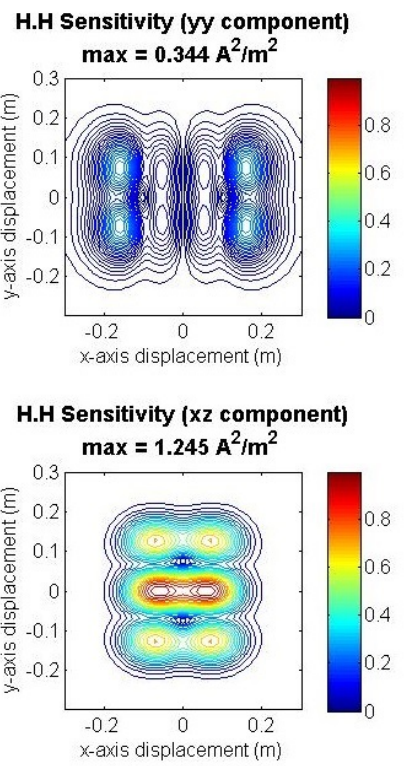

Normalisation Factor: $100 \%=1.286 \mathrm{~A}^{2} / \mathrm{m}^{2}$
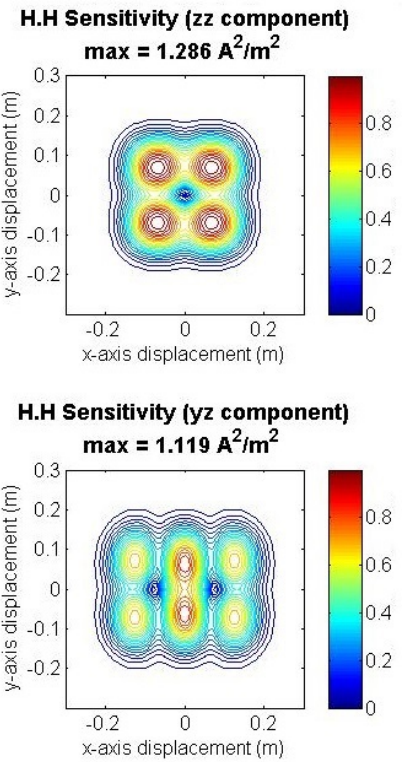

Fig. 4. Simulated sensitivity map for coil geomtery at a distance of $0.1 \mathrm{~m}$

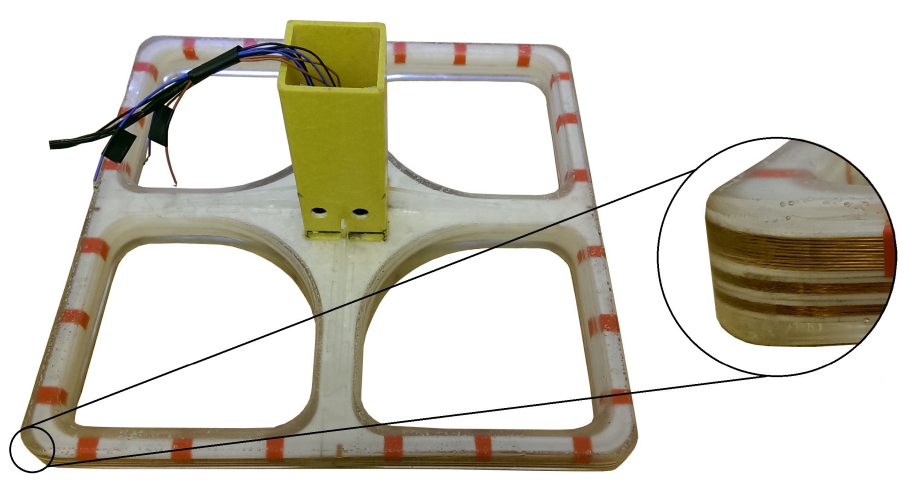

Fig. 7. Encapsulated coil array

the transmit coil at the top, followed by two layers of receive coils. High gain front-end circuitry is embedded within the yellow GRP pole, onto which the sensor is mounted.

\section{CONClusions And Future Work}

This paper has presented a method by which it is possible to evaluate the effectiveness of a specified coil, or array of coils, at inverting the magnetic polarisability tensor. Although specific examples have been used, the methods are generalisable to any design. For the design presented it has been shown that the estimated mean tensor error that should be expected between $10 \mathrm{~dB}$ and $20 \mathrm{~dB}$ SNR is in the region of $<6 \%$, with a standard deviation $<25 \%$.

The next step for this research is to connect the system to a suitable data acquisition system, and perform measurements to verify the forward and inverse modelling presented in this paper. This will be carried out by mounting the scanner onto an $\mathrm{x}, \mathrm{y}, \mathrm{z}$ plotter, and scanning the coils over targets with known responses.

\section{ACKNOWLEDGMENTS}

The authors would like to thank Find A Better Way for their financial support of this research under the SEMIS programme. The authors would also like to thank John Davidson of the University of Manchester, and Christos Ktistis and Bachir Dekdouk, formerly of the University of Manchester, for their contribution to this work.

\section{REFERENCES}

[1] D. Guelle et al. Metal Detector Handbook For Humanitarian Demining Luxembourg, European Commission, 2003.

[2] R. Keeley Understanding Landmines and Mine Action Massachusetts Institute of Technology, USA, 2003.

[3] United Nations Assistance in Mine Clearance: Report of the SecretaryGeneral New York, United Nations, A/49/357 p. 4, 1994.

[4] P. Barron Report: Funding Needed for Mine Detectors Phnom Penh, The Cambodia Daily, 2003.

[5] D. Daniels et al. Using MINEHOUND in Cambodia and Afghanistan The Journal of ERW and Mine Action 18.2, p. 46-51, 2014.

[6] United Nations (1996,08/10/2014) Land Mine Facts. Available: http://www.un.org/Depts/dha/mct/facts.htm

[7] Marsh L A et. al. Three-dimensional object location and inversion of the magnetic polarisability tensor at a single frequency using a walk-through metal detector Meas. Sci. Technol. vol. 24045102 doi:10.1088/09570233/24/4/045102, 2013.

[8] Makkonen $\mathrm{J}$ et. al. KNN classification of metallic targets using the magnetic polarizability tensor Meas. Sci. Technol. vol. 25055105 doi:10.1088/0957-0233/25/5/055105, 2014.

[9] Numerical Algorithms Group (2012, 09/10/2014) NAG Toolbox for Matlab nag_opt_lsq_uncon_mod_func_easy (e04fy) Available: http://www.nag.co.uk/numeric/MB/manual_23_1/pdf/E04/e04fy.pdf

[10] Numerical Algorithms Group (2014, 09/10/2014) The NAG Toolbox for MATLAB - Numerical Algorithms Group Available: http://www.nag.co.uk/numeric/MB/start.asp

[11] EOS GmbH - Electro Optical Systems (2008, 07/10/2014) Material data sheet - PA2200. Available: https://www.shapeways.com/rrstatic/material_docs/mds-strongflex.pdf 